\title{
Using a finite element grid on corner points in flow models
}

\section{J. Matthews R. D. Braddock* G. C. Sander ${ }^{\dagger}$}

(Received 11 July 2001; revised 29 November 2002)

\begin{abstract}
One of the main functions of a multilayer cover liner is to prevent water from infiltrating into mine or other waste thereby preventing the occurrence of ground water pollution. In the past, numerical models have predominantly dealt with vertical infiltration or infiltration into sloping hillsides of infinite extent. The two layer model investigated in this paper has a more realistic shape which is piece-wise linear with a horizontal top, vertical bottom and a sloping section inbetween. At the intersection of these segments are corner points where there are changes from sloping flow dynamics to either vertical or horizontal flow dynamics, depending on the corner point. The abrupt change in dynamics at the corner points can cause numerical problems especially when
\end{abstract}

${ }^{*}$ Griffith University, Brisbane, Australia.

mailto:c.matthews@griffith. edu.au

${ }^{\dagger}$ Loughborough University, Leistershire, UK.

${ }^{0}$ See http://anziamj . austms . org. au/V44/CTAC2001/Matt for this article, (C) Austral. Mathematical Soc. 2003. Published 1 April 2003. ISSN 1446-8735 
dealing with the boundary condition at the interface of two soils. This paper will deal with the corner point problem at the soil layer interface and, in particular, investigate the use of a finite element grid around the corner points.

\section{Contents}

1 Introduction

2 Corner point problem on the interface boundary

3 Using a finite element grid at the corner point

4 Handling discontinuities across the interface boundary

C539

5 Results

C543

6 Conclusion

C547

References

C548

\section{Introduction}

When a waste dump reaches its full capacity a cover liner is employed as a last measure to isolate the potentially harmful waste from the environment. One of the main objectives of a cover liner is to prevent water from infiltrating into the waste and thereby preventing any additional risk of groundwater pollution. In the past, cover liners have been predominantly made from compacted clays and/or geosynthetic materials making use of their very low hydraulic 
conductivity properties. However, these kinds of cover liners have not always been successful and their appropriateness has been questioned $[2,7]$. In recent times, cover liners made from various soil layers have been investigated. These multilayered liners rely on the diversion capacities of capillary barriers, which are fine over coarse soil structures $[8,11]$. Nevertheless, there is still a need to understand flow dynamics through such soil structures so as to maximise their ability to divert water away from underlying waste.

Numerical models have been used as a tool, by several researchers, to understand flow dynamics through cover liner systems. In the past, numerical models have predominantly dealt with infiltration into vertical soil profiles or sloping soil profiles of infinite extent [3, 13, 8]. The model presented in this paper has a more realistic shape being piece-wise linear with a horizontal top, vertical edges near the bottom and a sloping section in-between (Figure 1). During the simulation, the top boundaries A-B-C experience a constant rainfall flux while boundaries A-H and C-D are assigned a no flow condition as a line of symmetry. The no flow condition along C-D assumes that another waste dump butts up against the boundary C-D. A no flow condition is also applied to the boundary immediately above the waste (E-F-G-H) and boundary D-E allows infiltrating water to drain naturally from the system. Boundary $\mathrm{A}^{\prime}-\mathrm{B}^{\prime}-\mathrm{C}^{\prime}-\mathrm{D}^{\prime}$ is the soil layer interface separating the top soil (Soil 1) from the bottom soil (Soil 2).

To model unsaturated flow through the cover liner (Figure 1), Schiesser's Method of Lines [12] was used to solve the following two dimensional $h$-based version of Richard's Equation:

$$
C(h) \frac{\partial h}{\partial t}=\frac{\partial}{\partial x}\left(K(h) \frac{\partial h}{\partial x}\right)+\frac{\partial}{\partial z}\left(K(h) \frac{\partial h}{\partial z}\right)-\frac{\partial K}{\partial z},
$$

where $C(=\partial \theta / \partial h)$ is the water capacity, $\theta$ is water content, $h$ is hydraulic pressure, $K$ is the hydraulic conductivity, $x$ is the horizon- 


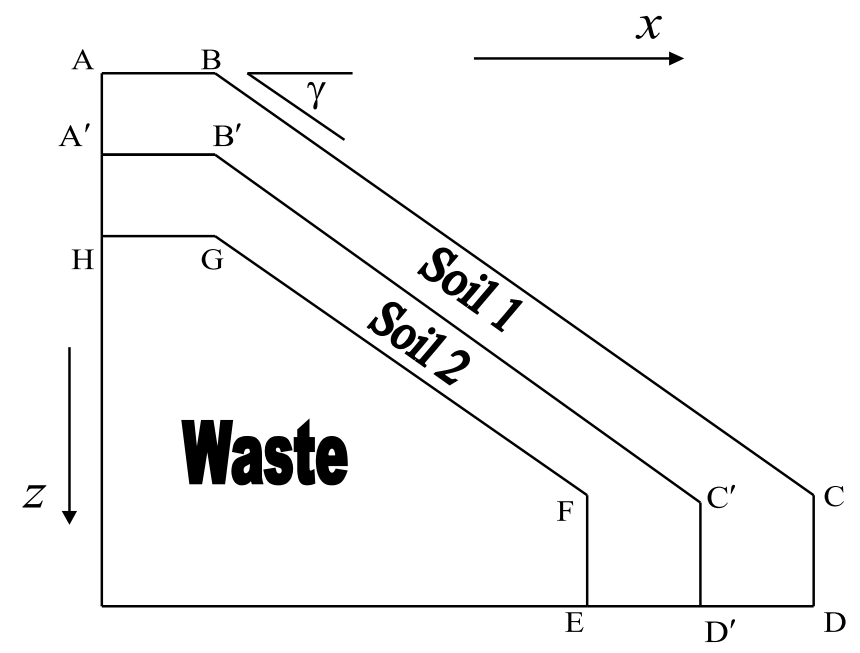

FiguRE 1: Piece-wise linear cover liner model

tal coordinate positive to the right and $z$ is the vertical coordinate positive downwards. The $\theta$ - $h$ relationship (main wetting curve) and the hydraulic functions $K$ and $C$ evaluated using van-Genuchten functions for two soils, Glendale clay (Soil 1) and Berino loamy fine sand (Soil 2) [4]. The $h$-based version is usually chosen to describe layered soil systems since $\theta$ is generally discontinuous between the two soils. In addition, Richard's Equation is based on the conservation of mass, where flux in the $z$ and $x$-direction is given by Darcy's Law:

$$
q_{z}=-K\left(\frac{\partial h}{\partial z}-1\right) \quad \text { and } \quad q_{x}=-K \frac{\partial h}{\partial x} \quad \text { respectively }
$$

Since there is a direct relationship between $\theta$ and $h$, Richard's equation can be written in terms of $\theta$ or $h$ by a simple change of variable. From Equation (1), it is evident that change in flux may 
be written in terms of $\partial \theta / \partial t$ since

$$
C(h) \frac{\partial h}{\partial t}=\frac{\partial \theta}{\partial t} .
$$

Schiesser's Method of Lines reduces Equation (1) to a set of ordinary differential equations (ODEs) by discretising the spatial part of the equation. The discretisation technique used by Schiesser is based on a finite differencing scheme consisting of upwinding and downwinding differencing to account for the domain boundaries. The vector $\mathbf{h}$ contains the nodal values of the hydraulic pressure at the nodes of the finite difference lattice. Boundary conditions are not directly incorporated into the finite differencing scheme but are imposed on $\mathbf{h}$ or $\partial \mathbf{h} / \partial z$ distribution vectors depending on the type of boundary condition [12]. The resultant set of oDEs is of the form

$$
\frac{\partial \mathbf{h}}{\partial t}=\mathbf{f}(\mathbf{h}, t), \quad \mathbf{h}(z, x, 0)=\mathbf{h}_{0},
$$

where bold face represents a vector, $\mathbf{f}(\mathbf{h}, t)$ is the resultant vector from applying the finite differencing scheme to the spatial derivatives in Equation (1) and $\mathbf{h}_{0}$ is the initial $h$ distribution vector. Equation (4) can be integrated by any conventional ODE integrator. A full account of applying the Method of Lines to the cover liner problem is given in [5]. Note that the non-linear term $C(h)$ is evaluated at each node and divided through Equation (1), becoming part of $\mathbf{f}(\mathbf{h}, t)$ in Equation (4), to gain an expression for $\partial \mathbf{h} / \partial t$.

\section{Corner point problem on the interface boundary}

The main difficulty in solving Richard's Equation over the domain depicted in Figure 1, is providing a physically accurate solution 
around the corner points situated at the interface between the two soils $\left(\mathrm{B}^{\prime}\right.$ and $\left.\mathrm{C}^{\prime}\right)$. At the interface, the two conditions of conservation of the normal flux and hydraulic pressure must hold $[4,11]$ :

$$
\begin{aligned}
& q_{1}=q_{2}, \\
& h_{1}=h_{2},
\end{aligned}
$$

where the subscript denotes soil type. These interface conditions are applied on the horizontal $\left(\mathrm{A}^{\prime}\right.$ and $\left.\mathrm{B}^{\prime}\right)$, sloping $\left(\mathrm{B}^{\prime}\right.$ and $\left.\mathrm{C}^{\prime}\right)$, and vertical $\left(\mathrm{C}^{\prime}\right.$ and $\left.\mathrm{D}^{\prime}\right)$ sections of the interface boundary. Considering each interface boundary separately, Equation (5) is rewritten using Darcy's Law (2), and equating normal fluxes for the horizontal, sloping and vertical boundaries respectively:

$$
\begin{gathered}
K_{1}\left(1-\frac{\partial h_{1}}{\partial z}\right)=K_{2}\left(1-\frac{\partial h_{2}}{\partial z}\right), \\
K_{1}\left(\cos \gamma+\sin \gamma \frac{\partial h_{1}}{\partial x}-\cos \gamma \frac{\partial h_{1}}{\partial z}\right) \\
=K_{2}\left(\cos \gamma+\sin \gamma \frac{\partial h_{2}}{\partial x}-\cos \gamma \frac{\partial h_{2}}{\partial z}\right), \\
-K_{1} \frac{\partial h_{1}}{\partial x}=-K_{2} \frac{\partial h_{2}}{\partial x},
\end{gathered}
$$

where $\gamma$ is the angle of slope as depicted in Figure 1. To satisfy Equation (6), Equations (7), (8) and (9) can be solved iteratively for a given $h$ on their respective boundaries. However, as both boundaries merge into the corner point $\mathrm{B}^{\prime}$ or $\mathrm{C}^{\prime}$, Equations (7) and (8) and Equations (8) and (9) must be resolved on their respective corner points on a single nodal point within the system. This implies that there are three conditions to be satisfied by the two values, of $h_{1}$ and $h_{2}$, at each of the points $\mathrm{B}^{\prime}$ and $\mathrm{C}^{\prime}$, and the system is overdetermined. Instead, the continuity of the mass of water at each corner 
point is represented in terms of finite elements. The aim of this paper is to investigate the use of a finite element patch at each corner point, to satisfy the conservation of water at $\mathrm{B}^{\prime}$ and $\mathrm{C}^{\prime}$, and hence to estimate $h_{1}$ and $h_{2}$ at the corner points.

\section{Using a finite element grid at the corner point}

In Finite Elements, a combination of interpolation and integration is used to obtain an expression for total mass within an element or grid system [1]. Essentially, the technique provides a weighting for each node within a grid that can be applied to a variable $(v)$ giving an approximation of the integral of $v$ over the grid. For example, consider the grid system from the cover liner model around the point $\mathrm{B}^{\prime}$ as depicted in Figure 2 where points 1, 2, 3 and 6 are in Soil 1, points 4, 5, 7, 8 and 9 are in Soil 2. In addition, the step sizes $\Delta x$ and $\Delta z$ are linked by the relationship $\Delta z=\Delta x \tan \gamma$, which ensures that nodal points lie along the sloping boundaries.

Using quadratic interpolation and integrating for each node [1] yields the following nine-point weighting scheme:

$$
\begin{gathered}
\iint_{x} v d x d z=\frac{4 \Delta x \Delta z}{36}\left(v_{1}^{1}+4 v_{2}^{1}+v_{3}^{1}+4 v_{4}^{2}+16 v_{5}^{2}+4 v_{6}^{1}\right. \\
\left.+v_{7}^{2}+4 v_{8}^{2}+v_{9}^{2}\right)
\end{gathered}
$$

where $v$ is a variable of interest, subscripts denotes node number from Figure 2, superscripts denotes soil type, and $4 \Delta x \Delta z$ is the area of the grid system.

To conserve the volume of water around the corner point by letting $v=\partial \theta / \partial t$, Equation (10) is used to derive an approximation 


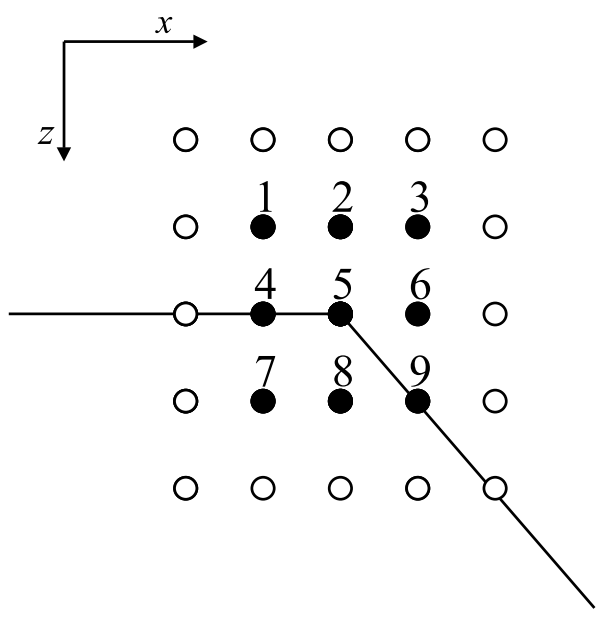

Figure 2: Grid system around the top corner point $\left(\mathrm{B}^{\prime}\right)$

for the change in water content within the total area of the grid. Using the law of conservation of mass, the total change in water content is equated to the difference of the flux moving in and out of the grid:

$$
\begin{gathered}
\frac{4 \Delta x \Delta z}{36}\left(\left.\frac{\partial \theta}{\partial t}\right|_{1} ^{1}+\left.4 \frac{\partial \theta}{\partial t}\right|_{2} ^{1}+\left.\frac{\partial \theta}{\partial t}\right|_{3} ^{1}+\left.4 \frac{\partial \theta}{\partial t}\right|_{4} ^{2}+\left.16 \frac{\partial \theta}{\partial t}\right|_{5} ^{2}\right. \\
\left.+\left.4 \frac{\partial \theta}{\partial t}\right|_{6} ^{1}+\left.\frac{\partial \theta}{\partial t}\right|_{7} ^{2}+\left.4 \frac{\partial \theta}{\partial t}\right|_{8} ^{2}+\left.\frac{\partial \theta}{\partial t}\right|_{9} ^{2}\right)=\Delta Q
\end{gathered}
$$

where $\Delta Q=Q_{\text {in }}-Q_{\text {out }}$. Since flux is defined in the direction of the $z$ and $x$-axis, $Q_{\text {in }}$ is calculated by numerically integrating $q_{z}$ across the top of the grid (nodes $\{1,2,3\}$ ), and $q_{x}$ over the left-hand side of the grid (nodes $\{1,4,7\}$ ). Similarly, $Q_{\text {out }}$ is calculated by numerically integrating $q_{z}$ and $q_{x}$ over the bottom (nodes $\{7,8,9\}$ ) and right-hand side (nodes $\{3,6,9\}$ ) of the grid respectively. Equation (11) is rearranged to obtain an expression in terms of $\partial \theta / \partial t$ on 
the corner point (Node 5) by using Equation (3):

$$
\begin{aligned}
\left.\frac{\partial \theta}{\partial t}\right|_{5} ^{2}= & \frac{1}{16}\left[\frac{36 \Delta Q}{4 \Delta x \Delta z}-\left(\left.\frac{\partial \theta}{\partial t}\right|_{1} ^{1}+\left.4 \frac{\partial \theta}{\partial t}\right|_{2} ^{1}+\left.\frac{\partial \theta}{\partial t}\right|_{3} ^{1}\right.\right. \\
& \left.\left.+\left.4 \frac{\partial \theta}{\partial t}\right|_{6} ^{1}+\left.4 \frac{\partial \theta}{\partial t}\right|_{4} ^{2}+\left.\frac{\partial \theta}{\partial t}\right|_{7} ^{2}+\left.4 \frac{\partial \theta}{\partial t}\right|_{8} ^{2}+\left.\frac{\partial \theta}{\partial t}\right|_{9} ^{2}\right)\right]
\end{aligned}
$$

The above formulation does not provide a good estimate of $\partial h / \partial t$ at the corner point since discontinuities in $\partial \theta / \partial t, q_{x}$ and $q_{z}$, which exist across the interface boundary, have not been considered. These discontinuities effect both the Finite Element Grid formulation as well as the estimate of $\triangle Q$ within the grid. In particular, Node 9 in Figure 2 needs to account for discontinuities in $q_{z}$ from integrating over the right-hand side of the grid and $\partial \theta / \partial t$ from the finite element grid formulation. A discontinuity in $q_{z}$ may also exists across the interface but is avoided by assuming Node 9 is in Soil 2. However, it is desirable to account for this jump in $q_{z}$ to provide a more accurate estimate of $\Delta Q$ for the grid around the corner point.

\section{Handling discontinuities across the interface boundary}

To handle the discontinuities across the interface boundary, a new slightly expanded grid system will be introduced to the interface corner points. The new grid for the top corner point at $\mathrm{B}^{\prime}$ is illustrated in Figure 3. As part of the new grid system, interdomain fictitious points are placed along the interface boundary and are given the hydraulic properties of Soil 1. In essence, two sets of nodes lie along the interface allowing both soils to be represented 


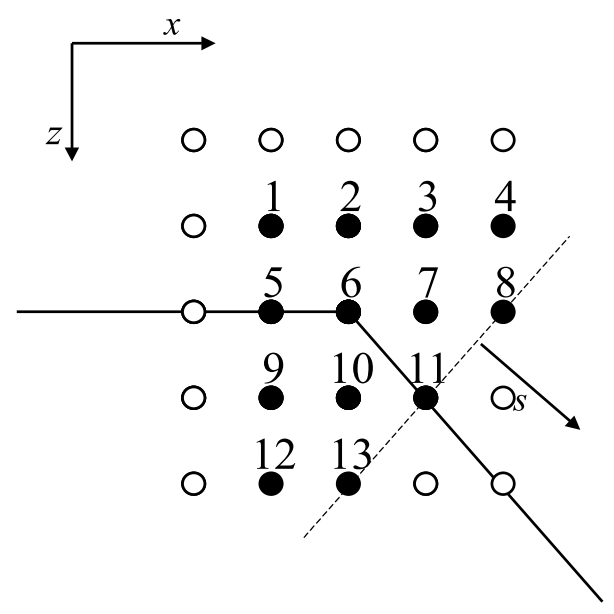

Figure 3: New grid system around the top corner point $\left(\mathrm{B}^{\prime}\right)$ to assist in handling discontinuities

on the boundary. The two sets of nodes enables variables to be integrated up to the interface boundary, in both soils, minimising any integration error from discontinuities. In addition, a sloping face is introduced (nodes $\{8,11,13\}$ ) to handle the three discontinuities at Node 11 (corresponding to Node 9 in Figure 2). Flux across the sloping face is calculated in the $s$-direction, which is perpendicular to the sloping face:

$$
q_{s}=q_{z} \cos \gamma+q_{x} \sin \gamma
$$

Since $\Delta z$ and $\Delta x$ is related by $\tan \gamma$, the direction of the $s$ coordinate is only parallel to the sloping surface of the cover liner when $\gamma=45^{\circ}$.

The finite element formulation for the new grid will consist of a series of 4-point linear interpolated rectangles and 3-point linear interpolated triangles. These schemes have an evenly distributed weighting for each node of $\Delta x \Delta z / 4$ and $\Delta x \Delta z / 6$ respectively when 
considering the area of the grid elements in Figure 3. The two weighting schemes are applied to the grid system in a step-wise manner for each soil separately making use of the fictitious points in Soil 1. The resultant equation for each soil is then added together to obtain a complete estimate for the change in water content, $\theta$ with respect to time, within the grid. For example the formulation for top corner point is

$$
\iint_{\text {Grid }} \frac{\partial \theta}{\partial t} d x d z=\left.\iint_{\text {Grid }^{1}} \frac{\partial \theta}{\partial t}\right|^{1} d x d z+\left.\iint_{\text {Grid }^{2}} \frac{\partial \theta}{\partial t}\right|^{2} d x d z
$$

where

$$
\begin{aligned}
& \left.\iint_{\text {Grid }^{1}} \frac{\partial \theta}{\partial t}\right|^{1} d x d z=\Delta x \Delta z\left(\left.\frac{1}{4} \frac{\partial \theta}{\partial t}\right|_{1} ^{1}+\left.\frac{1}{2} \frac{\partial \theta}{\partial t}\right|_{2} ^{1}+\left.\frac{1}{4} \frac{\partial \theta}{\partial t}\right|_{3} ^{1}\right. \\
& \left.+\left.\frac{1}{4} \frac{\partial \theta}{\partial t}\right|_{4} ^{1}+\left.\frac{1}{4} \frac{\partial \theta}{\partial t}\right|_{5} ^{1}+\left.\frac{2}{3} \frac{\partial \theta}{\partial t}\right|_{6} ^{1}+\left.\frac{5}{6} \frac{\partial \theta}{\partial t}\right|_{7} ^{1}+\left.\frac{5}{12} \frac{\partial \theta}{\partial t}\right|_{8} ^{1}+\left.\frac{1}{3} \frac{\partial \theta}{\partial t}\right|_{11} ^{1}\right) \\
& \left.\iint_{\text {Grid }^{2}} \frac{\partial \theta}{\partial t}\right|^{2} d x d z=\Delta x \Delta z\left(\left.\frac{1}{4} \frac{\partial \theta}{\partial t}\right|_{5} ^{2}+\left.\frac{5}{12} \frac{\partial \theta}{\partial t}\right|_{6} ^{2}+\left.\frac{1}{2} \frac{\partial \theta}{\partial t}\right|_{9} ^{2}\right. \\
& \left.+\left.\frac{2}{3} \frac{\partial \theta}{\partial t}\right|_{10} ^{2}+\left.\frac{1}{3} \frac{\partial \theta}{\partial t}\right|_{11} ^{2}+\left.\frac{1}{4} \frac{\partial \theta}{\partial t}\right|_{12} ^{2}+\left.\frac{5}{12} \frac{\partial \theta}{\partial t}\right|_{13} ^{2}\right)
\end{aligned}
$$

superscripts denotes soil type, and subscripts denotes node number (Figure 3). In the above formulation, the rectangular scheme was applied to nodes $\{1,2,5,6\},\{2,3,6,7\},\{3,4,7,8\}$ in Soil 1 and $\{5,6,9,10\},\{9,10,12,13\}$ in Soil 2 and the triangular scheme was applied to nodes $\{6,7,11\},\{7,8,11\}$ in Soil 1 and $\{6,10,11\}$, $\{10,11,13\}$ in Soil 2. In addition, the fictitious points are nodes $\{5,6,11\}$ in Soil 1 , therefore, the actual interface boundary is represented by nodes $\{5,6,11\}$ in Soil 2 . 
To calculate the amount of flux entering and leaving the grid system, a trapezoid rule is applied to the top (nodes $\{1,2,3,4\}$ ), bottom (nodes $\{12,13\}$ ), left (nodes $\{1,5,9,12\}$ ) and right (nodes $\{4,8,11,13\})$ edges of the grid:

$$
\begin{aligned}
Q_{t} & =\frac{\Delta x}{2}\left(\left.q_{z}\right|_{1} ^{1}+\left.2 q_{z}\right|_{2} ^{1}+\left.2 q_{z}\right|_{3} ^{1}+\left.q_{z}\right|_{4} ^{1}\right), \\
Q_{b} & =\frac{\Delta x}{2}\left(\left.q_{z}\right|_{12} ^{1}+\left.q_{z}\right|_{13} ^{1}\right) \\
Q_{l} & =\frac{\Delta z}{2}\left(\left.q_{x}\right|_{1} ^{1}+\left.q_{x}\right|_{5} ^{1}\right)+\frac{\Delta z}{2}\left(\left.q_{x}\right|_{5} ^{2}+\left.2 q_{x}\right|_{9} ^{2}+\left.q_{x}\right|_{12} ^{2}\right) \\
Q_{r} & =\frac{\Delta z}{2}\left(\left.q_{x}\right|_{4} ^{1}+\left.q_{x}\right|_{8} ^{1}\right)+\frac{\Delta s}{2}\left(\left.q_{s}\right|_{8} ^{1}+\left.q_{s}\right|_{11} ^{1}\right)+\frac{\Delta s}{2}\left(\left.q_{s}\right|_{11} ^{2}+\left.q_{s}\right|_{13} ^{2}\right)
\end{aligned}
$$

where $q_{z}, q_{x}$ and $q_{s}$ are the flux in the $z, x$ and $s$-direction respectively (Figure 3) and $\Delta s=\sqrt{\Delta z^{2}+\Delta x^{2}}$ is the step size in the $s$-direction. At Node 5 , a discontinuity in $q_{x}$ can exist across the interface, which is accounted for in $Q_{l}$ (Equation (15)) by numerically integrating $q_{x}$ up to Node 5 in each soil separately. Similarly, $Q_{l}$ highlights the possible discontinuity in $q_{s}$ across the sloping surface at Node 11 integrating up to Node 11 in both soils. In essence, handling the discontinuity in the form of $q_{s}$ on a sloping surface accounts for the possible discontinuities in $q_{x}$ and $q_{z}$ at this node (Equation (13)).

The total change in flux within the grid is therefore

$$
\Delta Q=Q_{t}-Q_{b}+Q_{l}-Q_{r}
$$

Equating the Finite Element formulation with Equation (16) and 
rearranging for $\partial \theta / \partial t$ at the corner point yields

$$
\begin{aligned}
& \left.\frac{\partial \theta}{\partial t}\right|_{6} ^{2}=\frac{12}{5}\left\{\frac{\Delta Q}{\Delta x \Delta z}-\left(\frac{1}{\Delta x \Delta z}\left[\left.\iint_{\text {Grid }^{1}} \frac{\partial \theta}{\partial t}\right|^{1} d x d z\right]+\left.\frac{1}{4} \frac{\partial \theta}{\partial t}\right|_{5} ^{2}+\right.\right. \\
& \left.\left.+\left.\frac{1}{2} \frac{\partial \theta}{\partial t}\right|_{9} ^{2}+\left.\frac{2}{3} \frac{\partial \theta}{\partial t}\right|_{10} ^{2}+\left.\frac{1}{3} \frac{\partial \theta}{\partial t}\right|_{11} ^{2}+\left.\frac{1}{4} \frac{\partial \theta}{\partial t}\right|_{12} ^{2}+\left.\frac{5}{12} \frac{\partial \theta}{\partial t}\right|_{13} ^{2}\right)\right\}
\end{aligned}
$$

Equation (17) is then evaluated in terms of $\partial h / \partial t$ by using Equation (3) resulting in an ODE that can be including into the ODE system (Equation (4)) for the cover liner model.

\section{$5 \quad$ Results}

Two test cases are considered to test the performance of the finite element grid for the interface corner points. For the first test case, both soils are assigned the same hydraulic properties creating a pseudo-single layer cover liner model. In other words, the interface boundary should not affect the behaviour of the infiltrating water. The second test case will be to test the model on a fine over coarse soil structure or capillary barrier. A capillary barrier holds water at the soil layer interface by capillary forces inhibiting the flow of water into the coarse soil. Also, when a capillary barrier is inclined, water flows laterally down the slope above the interface. Therefore, at the interface corner points, both flow dynamics should be evident with the corner point acting as a transitional point between the two flow dynamics.

For all test cases, the waste dump is 1 metre high with a slope $(\gamma)$ of $30^{\circ}$. The top flat section $\left(\mathrm{A}^{\prime}-\mathrm{B}^{\prime}\right)$ and the total depth of the cover liner (A-H) is $30 \mathrm{~cm}$ long with a depth of $12 \mathrm{~cm}$ for Soil 1 (A$\mathrm{A}^{\prime}$ ). Lengths $\mathrm{C}-\mathrm{D}$ and $\mathrm{E}-\mathrm{D}$ are related to A-B and A-H respectively 
by $\tan \gamma$ in a similarly fashion to the step sizes $\Delta x$ and $\Delta z$. The step size $\Delta z$ was set at $1 \mathrm{~cm}$ resulting in a $\Delta x$ of approximately $1.732 \mathrm{~cm}$ and 3193 as the total number of nodes in the system. The waste dump was chosen to be small in dimension so that a wide variety of flow dynamics could be observed in a relatively short simulation period. In addition, the initial condition is given a uniform distribution of $h=-100 \mathrm{~cm}$, the surface of the cover liner is given a moderate rainfall rate equal to half of the saturated hydraulic conductivity of the top soil and the bottom flat boundary (D-E) is assigned a no flow condition. The latter boundary condition will ensure that an accurate mass balance can be calculated to determine whether water is being lost or introduced into the system by the numerical solution. For reason of brevity, the solution is only shown for the top interface corner point.

For the first test case, the model was run for a simulation time of 4.8 hours using the soil properties of Glendale clay [4], resulting in the contour plots of the hydraulic pressure $(h)$ presented in Figure 4. In Figure 4, the black solid line represents the interface boundary between Soil 1 and Soil 2. It is evident from Figure 4 that the interface has successful decomposed to a single layer problem having no visible effect on behaviour of the infiltrating water. This also suggests that the Finite Element Grid is preserving mass around the corner point and the ODE, which resulted from the finite element grid formulation, successfully models the transient behaviour at the corner point. This is further reinforced by a mass balance error of approximately $0.077 \%$. The mass balance was calculated by integrating $\theta$ across each soil layer, using the fictitious points to maintain accuracy. Simpsons rule was used, in conjunction with the repeated one dimensional integration technique, for handling surface integrals [10]. In addition, the result from the first test case was compared against an actual one layer cover liner model [6] calculating relative error for each node within the cover liner system. 


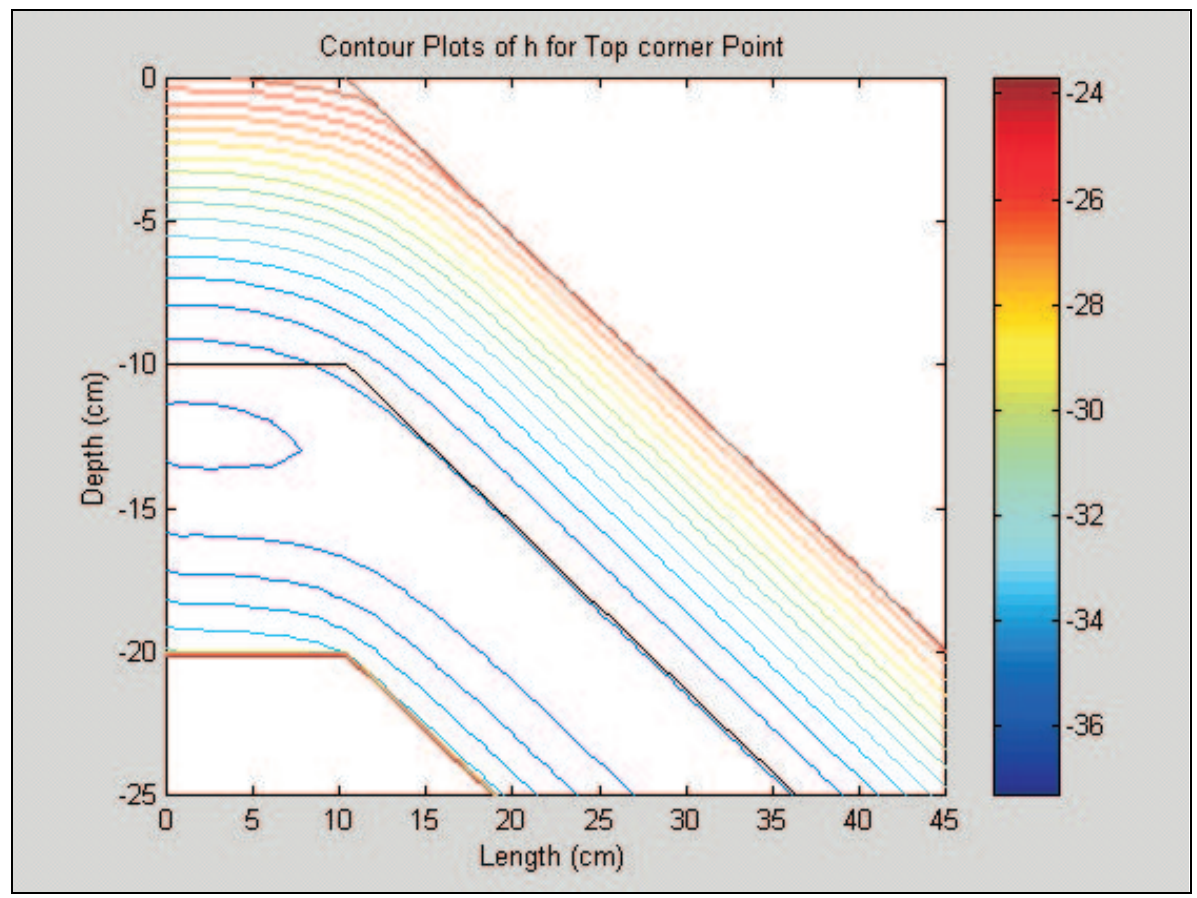

Figure 4: Contours plots of hydraulic pressure $(h)$ for Test Case 1

Using Simpson's Rule, total relative error over the whole domain was estimated at 3.27 , which corresponds to an average relative error of $1.97 \times 10^{-4}$. The relative error on both corner points was calculated at $8.30 \times 10^{-4}$ and $2.19 \times 10^{-2}$ for the top and bottom interface corner point respectively.

The second test case (capillary barrier) was also run over a simulation time of 4.8 hours using Glendale clay for Soil 1 and Berino loamy fine sand for Soil 2 [13]. To give an idea of the contrast between the two soils, the saturated hydraulic conductivity for each soil are $13.1 \mathrm{~cm} /$ day and $541 \mathrm{~cm} /$ day respectively. The result from Test Case 2 is presented as contour plots of $h$ in Figure 5. Compar- 


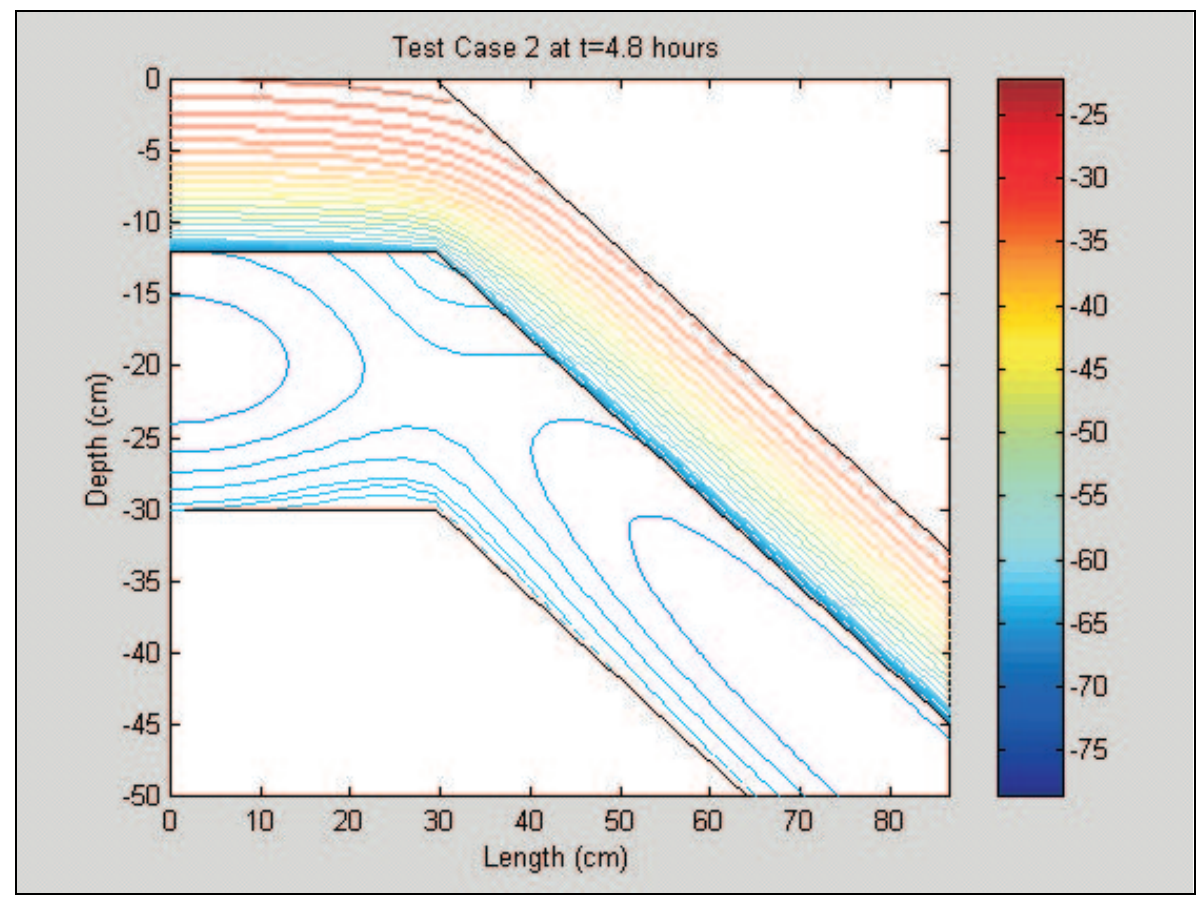

Figure 5: Contour plots of hydraulic pressure $(h)$ for Test Case 2

ing Figure 5 with Figure 4 highlights the effect of the capillary barrier with the flow of water being impeded by the interface boundary resulting in a gathering of water in Soil 1. As expected, infiltration from the top flat $\left(\mathrm{A}^{\prime}-\mathrm{B}^{\prime}\right)$ and the sloping surface $\left(\mathrm{B}^{\prime}-\mathrm{C}^{\prime}\right)$ away from the corner point is parallel to their respective surfaces [9]. Around the corner point the contours show a smooth transition between the horizontal and sloping flow dynamics, which suggests that the finite element formulation is working. Underneath the interface in Soil 2, redistribution occurs with water moving vertical downwards and laterally down the slope under gravitational forces. Lateral flow is more predominant in Soil 2 because of the coarseness and the higher hydraulic conductivity properties of the soil. The strength of the 
lateral flow is depicted by the splitting of the contours with a region of water sitting on the horizontal surface $(\mathrm{H}-\mathrm{G})$ and a region moving down the sloping. Along the interface boundary including the corner point, the contours show that $h$ remains continuous across the interface confirming that the boundary is satisfying Equation (6). As an additional test, a mass balance was performed resulting in a mass balance error of $0.123 \%$. The mass balance error is slightly higher than the previous test case, which is to be expected since the model must handle the contrast between the two soils.

\section{Conclusion}

For Test Case 1, the finite element formulation around the top corner point seems to conserves the amount of water flowing through the grid system. The bottom corner point does show a much larger relative error when compared against a one layer case but this can be attributed to the linear nature of the finite element formulation used in this study. Therefore, different finite element formulations should be experimented with using Test Case 1 as a litmus test. In both cases, the behaviour of infiltrating water exhibits a range of expected behaviour suggesting that the finite element grid technique works. This result is confirmed by the mass balance results, which show that mass is conserved within the system.

Acknowledgements: I thank Dr John Norbury, Oxford University for his valuable input and guidance. 


\section{References}

[1] Akin, J. ED., Finite Element Analysis for Undergraduates, Academic Press Inc. (San Diego), 1988. C537

[2] Donald, S. B., and McBean, E. A., Statistical analyses of compacted clay landfill liners. Part 1: Model Development, Can. J. Civ. Eng., 21, 1994, 872-882. C533

[3] Fayer, M. J., Rockford, M. L., and Campbell, M. D., Hydrologic Modelling of Protective Barriers: Comparison of Field Data and Simulation Results, Soil Sci. Soc. Am. J., 56, 1992, 690-700. C533

[4] Hills, R. G., Porro, I., Hudson, D. B., and Wierenga, P. J., Modeling One-Dimensional Infiltration Into Very Dry Soils. Part 1: Model Development and Evaluation, Water Resour. Res., 25(6), 1989, 1259-1269. C534, C536, C544

[5] Matthews, C. J., Modelling Water Flow through a Multi-layered Cover Liner for a Waste Dump, PhD thesis, Faculty of Environmental Sciences, Griffith University, 2002. C535

[6] Matthews, C. J., Braddock R. D., and Sander G. C., Modelling of a Cover Liner System for Waste Dumps, in Computational Techniques \& Applications: CTAC97, editors J. Noye, M. Teubner and A. Gill, World Scientific (Singapore), 1998, 401-408. C544

[7] Murray, G. B., McBean, E. A., Sykes, J. F., Estimation of Leakage Rates through Flexible Membrane Liners, Ground Water Monit. Remed., 15(4), 1995, 148-154. C533 
[8] Oldenburg, C. M., and Pruess, K., On Numerical Modeling of Capillary Barriers, Water Resour. Res., 29(4), 1993, 1045-1056. C533

[9] Philip, J. R., Hillslope infiltration: Planar slopes, Water Resour. Res., 27(1), 1991, 109-117. C546

[10] Press, W. H., Flannery, B. P., Teukolsky, S. A., and Vetterling, W. T., Numerical Recipes: The Art of Scientific Computing (Fortran Version), Cambridge University Press (Cambridge), 1989. C544

[11] Ross, B., The Diversion Capacity of Capillary Barriers, Water Resour. Res., 26(10), 1990, 2625-2629 C533, C536

[12] Schiesser, W., The Numerical Method of Lines: Integration of Partial Differential Equations, Academic Press Inc. (San Diego), 1991. C533, C535

[13] Yeh, T.-C., Guzman, A., Srivastava, R., and Gagnard, P. E., Numerical Simulation of the Wicking Effect in Liner Systems, Ground Water., 32(1), 1994, 2-11. C533, C545 